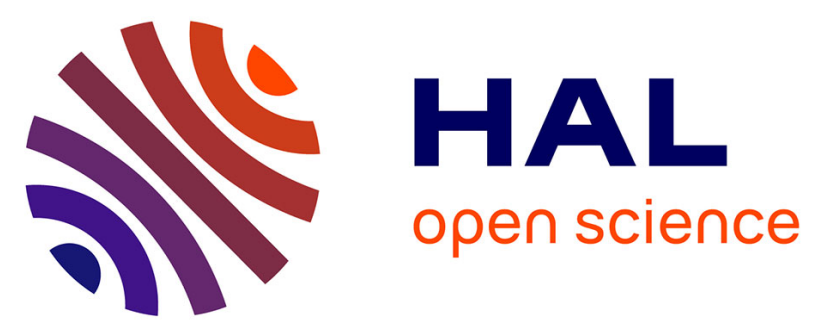

\title{
L'articulation des usages diagnostique et interactif d'un seul et même système de contrôle de gestion : le cas d'un système d'indicateurs environnementaux dans une entreprise française de vins et spiritueux
}

Angèle Renaud

\section{To cite this version:}

Angèle Renaud. L'articulation des usages diagnostique et interactif d'un seul et même système de contrôle de gestion: le cas d'un système d'indicateurs environnementaux dans une entreprise française de vins et spiritueux. Finance Contrôle Stratégie, 2013, 16 (3), 37 p. hal-00879214

\author{
HAL Id: hal-00879214 \\ https://hal.science/hal-00879214
}

Submitted on 1 Nov 2013

HAL is a multi-disciplinary open access archive for the deposit and dissemination of scientific research documents, whether they are published or not. The documents may come from teaching and research institutions in France or abroad, or from public or private research centers.
L'archive ouverte pluridisciplinaire HAL, est destinée au dépôt et à la diffusion de documents scientifiques de niveau recherche, publiés ou non, émanant des établissements d'enseignement et de recherche français ou étrangers, des laboratoires publics ou privés. 


\title{
L'articulation des usages diagnostique et interactif d'un seul et même système de contrôle de gestion : le cas d'un système d'indicateurs environnementaux dans une entreprise française de vins et spiritueux
}

\author{
Angèle RENAUD, \\ Maître de Conférences, Université de Bourgogne, IAE de Dijon, \\ Laboratoire d'Economie et de Gestion (LEG)
}

Classification JEL : M41, M14, Q5

Correspondance : angele.renaud@u-bourgogne.fr

Résumé :

En mobilisant le cadre conceptuel des leviers de contrôle de Simons (1995), cet article montre comment articuler les usages diagnostique et interactif d'un seul et même système de contrôle de gestion (en l'occurrence d'un système d'indicateurs environnementaux) pour permettre le déploiement et l'émergence de nouvelles stratégies. En opposition à la littérature, nous proposons une articulation «en parallèle» comme alternative à l'articulation «en glissement » des leviers de contrôle. Ces résultats sont obtenus à partir d'une étude de cas réalisée dans une entreprise française de vins et spiritueux sujette à de fortes incertitudes environnementales stratégiques.

Mots clés :

Systèmes de contrôle de gestion, contrôle diagnostique, contrôle interactif, indicateurs environnementaux, stratégie verte.

(*)Remerciements :

L'auteur remercie les réviseurs anonymes pour leurs remarques et suggestions constructives.

Finance-Contrôle-Stratégie, Vol. 16, N³, 2013 -Angèle RENAUD 


\title{
Articulation of Diagnostic and Interactive Uses of the Same Management Control System: The Case of Environmental Indicators System in a French Wine and Spirits Firm
}

\author{
Angèle RENAUD, \\ Maître de Conférences, Université de Bourgogne, IAE de Dijon, \\ Laboratoire d'Economie et de Gestion (LEG)
}

Classification JEL : M41, M14, Q5

Correspondance : angele.renaud@u-bourgogne.fr

\begin{abstract}
:
With the conceptual framework of control levers of Simons (1995), this article shows how to coordinate diagnostic and interactive uses of the same "management control system" (in this case a system of environmental indicators) for the deployment and the emergence of new strategies. In contrast to the literature, we propose an articulation "in parallel" of levers of control as alternative to sliding levers of control. These results are obtained from a case study in a French wine and spirits subject to large strategic environmental uncertainties.
\end{abstract}

Keys words:

Management control systems, diagnostic control, interactive control, environmental performance indicators, environmental strategy.

Finance-Contrôle-Stratégie, Vol. 16, $N^{\circ} 3,2013$-Angèle RENAUD 


\section{Introduction}

Depuis une vingtaine d'années, un courant de recherche se développe sur les interactions entre le contrôle de gestion et la stratégie. Ce nouveau courant est notamment marqué par les travaux de Robert Simons (1995), qui vont au-delà de la contingence classique selon laquelle la mission du contrôle de gestion est de garantir la mise en œuvre de la stratégie (rôle historique défini par Anthony 1988). En effet, Simons propose une nouvelle conception du contrôle de gestion dont le rôle ne se limite plus à la déclinaison de la stratégie mais contribue également à sa formulation. L'auteur met ainsi en exergue une situation d'influence réciproque entre ces deux disciplines. Il définit alors le contrôle de gestion moderne comme des «processus et procédures formels fondés sur l'information que les managers utilisent pour maintenir ou modifier certaines configurations des activités de l'organisation» (Simons 1991, p. 49). Dans cette conception moderne, il est question de leviers de contrôle dont disposent les managers (Bouquin 2004). En cohérence avec la stratégie, les systèmes formels ${ }^{1}$ de contrôle de gestion jouent deux rôles distincts : ils sont utilisés tantôt comme des leviers de «contrôle diagnostique », tantôt comme des leviers de «contrôle interactif».

Selon la conceptualisation de l'auteur, l'usage «diagnostique » des systèmes de contrôle de gestion permet de décliner la stratégie délibérée des dirigeants en surveillant la bonne application des facteurs clés de succès pendant que leur usage «interactif» sert à gérer les « incertitudes stratégiques » et à stimuler l'apprentissage organisationnel pour favoriser l'émergence de nouvelles stratégies. D'après l'auteur, les entreprises performantes sont celles qui arrivent à articuler ces deux modes d'usage pour permettre à la fois la mise en œuvre et la formulation des stratégies. Il considère à cet effet que les dirigeants ne peuvent pas utiliser de manière interactive plusieurs systèmes de contrôle de gestion, ceci dans le but d'éviter une dispersion de leur

\footnotetext{
${ }^{1}$ Simons (1995) réduit le contrôle de gestion à ses seuls dispositifs formels. Comme l'auteur, le contrôle «informel » ou «invisible » ne fera pas l'objet de notre étude. Les concepts de contrôles «diagnostique » et «interactif» ne s'appliquent ici qu'aux systèmes formels de contrôle de gestion mis en place par les dirigeants. De ce fait, dans cet article, les termes «contrôle» ou «systèmes de contrôle » renvoient systématiquement au contrôle de gestion «formel» et non pas au «contrôle organisationnel », qui est un ensemble plus vaste et dont le contrôle de gestion ne constitue qu'un élément.
} 
attention et de paralyser leur processus de réflexion. Autrement dit, si une entreprise dispose de (n) systèmes de contrôle de gestion (e.g. les processus formels de planification, de budgétisation, de contrôle des coûts, d'analyse de la concurrence, d'évaluation de la performance), un seul ferait l'objet d'un usage interactif tandis que les (n-1) autres systèmes de contrôle de gestion seraient utilisés de manière diagnostique.

C'est ainsi qu'apparaît la question de l'articulation des modes d'usage dans la littérature. Contrairement à Simons, des chercheurs comme Tuomela (2005), Widener (2007), Naro et Travaillé (2010) et Essid et Berland (2011) cherchent à comprendre les interactions entre ces deux leviers de contrôle en dépassant la dialectique contradictoire pour en explorer les éventuelles complémentarités ou apprentissages réciproques. Ces auteurs s'interrogent sur le passage d'un levier de contrôle à l'autre et proposent le concept de «glissement» pour expliquer leur articulation. Malgré leurs apports clarificateurs, ces travaux réduisent la question de l'articulation à une vision « successive » des leviers de contrôle.

Pour éviter le «one best way», il semble légitime de s'interroger sur une autre façon d'articuler ces usages. Cette question se pose notamment dans le cas où un seul et même système de contrôle de gestion est utilisé pour répondre «simultanément» à des objectifs contradictoires : le déploiement et l'émergence de nouvelles stratégies. C'est dans ce contexte que s'inscrit notre recherche et la problématique à laquelle nous tentons de répondre est la suivante : comment articuler les usages diagnostique et interactif d'un seul et même système de contrôle de gestion pour permettre à l'entreprise de fonctionner? Dans le cas présent, le système de contrôle de gestion étudié est un système d'indicateurs environnementaux. Au-delà de la connaissance sur les interactions entre les leviers de contrôle, l'intérêt de cette recherche est de contribuer à la compréhension de l'utilisation des systèmes de contrôle de gestion dits «ambidextres » pour concilier exploration et exploitation des savoirs (au sens de March 1991) ainsi que du rôle des acteurs (dirigeants, contrôleurs de gestion, fonctionnels de l'environnement) dans cette articulation.

La méthodologie de recherche retenue pour traiter cette problématique repose sur une étude de cas menée auprès d'une entreprise française du secteur des vins et spiritueux, dénommée «Maison de champagne » pour des raisons de confidentialité. Cette dernière, filiale d'un Groupe coté soumis à la loi NRE (nouvelles 
régulations économiques), évolue dans un contexte de fortes incertitudes environnementales stratégiques. Dans cette entreprise, nous avons examiné comment les dirigeants se servent d'un seul et même système d'indicateurs environnementaux pour piloter leur performance et rendre des comptes aux parties prenantes. Les résultats de cette étude nous permettent de tirer des enseignements d'ordre théorique, managérial et méthodologique.

L'article est structuré en quatre parties : la première présente le cadre conceptuel de la recherche; la seconde justifie et expose la méthodologie de la recherche; la troisième décrit les résultats et la dernière souligne les contributions-clés de la recherche, ses limites et perspectives futures.

\section{Les leviers de contrôle diagnostique/interactif comme cadre conceptuel de la recherche}

La problématique de la recherche est de comprendre l'articulation des usages diagnostique et interactif d'un seul et même système de contrôle de gestion, en l'occurrence un système d'indicateurs environnementaux. Pour cela, nous mobilisons le cadre théorique des leviers de contrôle de Simons (1995) et des travaux empiriques qui en découlent et qui portent sur les interactions entre les leviers de contrôle (1.1). À partir de cette revue de la littérature, une grille d'analyse est élaborée (1.2) pour examiner l'objet de la recherche (1.3).

\subsection{La revue de la littérature : de la dialectique contradictoire à la nécessaire complémentarité des leviers de contrôle}

Depuis les travaux précurseurs de Simons (1987, 1990, 1991, $1994,1995,2000)^{2}$ sur les influences réciproques entre la stratégie et le

\footnotetext{
${ }^{2}$ Outre les leviers de contrôle diagnostique et interactif, Simons (1995) propose deux autres leviers de contrôle: les systèmes de valeurs (ou croyances) et les systèmes de contraintes. Ces deux leviers servent à délimiter le domaine stratégique de l'entreprise, les uns en insufflant un idéal aux membres de l'entreprise et les autres en fixant des règles de conduite afin que les acteurs se comportent dans le sens attendu par les dirigeants. Le credo, la mission ou la vision d'entreprise sont des exemples de systèmes de valeurs. Les systèmes de contraintes sont souvent représentés par des codes de conduite, des guides de procédures, des chartes éthiques... Ces deux leviers ne seront pas abordés dans cette recherche, car nous nous intéressons spécifiquement aux instruments de mesure et de pilotage de la performance qui, eux, font l'objet d'un usage diagnostique ou interactif. Selon Simons (1991, p. 52, 1994, p. 172), il s'agit notamment des budgets, tableaux de bord, plans financiers et opérationnels, système de veille informationnelle, systèmes de gestion des compétences et de l'emploi...
} 
contrôle de gestion, ce dernier peut être appréhendé selon deux modalités d'usage. En effet, un système de contrôle de gestion peut être utilisé, soit de manière «diagnostique » pour déployer la stratégie délibérée des dirigeants, soit de manière «interactive» afin de contribuer à l'émergence de nouvelles stratégies. Ces deux usages, d'apparence contradictoire, suggèrent une interprétation dialectique des systèmes de contrôle de gestion. Nous exposons ci-après les caractéristiques qui conduisent à les opposer.

En premier lieu, leurs objectifs semblent paradoxaux. Le contrôle diagnostique veille à la conformité des buts et tend à préserver la routine organisationnelle tandis que le contrôle interactif cherche à briser cette routine en stimulant l'innovation. En effet, selon Simons, les systèmes de «contrôle diagnostique » contraignent l'innovation pour s'assurer de l'atteinte des objectifs : ce sont «des systèmes formels de feedback utilisés par les dirigeants pour surveiller les résultats organisationnels et corriger les déviations par rapport aux standards de performance fixés préalablement» (Simons 1994, p. 170). A contrario, les systèmes de « contrôle interactif » laissent plus de place à l'innovation. Ils sont utilisés pour gérer les «incertitudes stratégiques » (des menaces latentes ou des opportunités qui conditionnent la réalisation de la stratégie), encourager l'apprentissage organisationnel par l'exploration de nouveaux savoirs et favoriser l'émergence de nouvelles stratégies (Simons 1995, p. 7, p. 93).

En second lieu, l'implication des dirigeants et des fonctionnels du contrôle de gestion varie selon le mode d'usage. D'un côté, le contrôle diagnostique repose sur le principe du management par exception, «où l'intervention des dirigeants est limitée à la résolution de problèmes imprévus ou complexes» (Bouquin 2004, p. 81) alors que les fonctionnels jouent un rôle central dans la préparation et l'interprétation de l'information (Simons 1987). Toutefois, la participation des dirigeants n'est pas pour autant exclue du processus de contrôle diagnostique car, comme l'explique Simons (1995, p.70-71), «pour assurer la réalisation de leur stratégie, les dirigeants doivent personnellement négocier les buts avec leurs subordonnés. Ils ne délèguent pas la fixation des objectifs puisque ceux-ci sont essentiels à l'accomplissement de la stratégie ». De l'autre côté, à l'inverse du contrôle diagnostique, l'usage interactif nécessite "une implication régulière et personnelle des dirigeants dans les décisions de leurs subordonnés » lorsque celle des fonctionnels est plutôt limitée (ibid. p. 95). Toujours selon l'auteur (1987, p. 338-362), l'information générée 
par le processus interactif est un ordre du jour important et récurrent suivi par les hauts niveaux de direction. De plus, ce processus demande une attention fréquente de la part des managers opérationnels à tous les niveaux de l'organisation et, plus que les résultats, ce sont les efforts qui sont récompensés.

En dernier lieu, le processus de transmission des informations diffère d'un mode d'usage à l'autre. Dans le contrôle diagnostique, les données sont transmises par des procédures formelles de reporting pendant que le processus interactif repose sur un débat permanent autour des données, des hypothèses et des plans d'action. Ces informations sont interprétées et discutées en réunion avec les supérieurs, les subordonnés et les pairs. Pour Simons (2000, p. 219), le débat et le dialogue sont les marques de fabrique d'un système de contrôle interactif. Et plus précisément, c'est le caractère répétitif des échanges qui en font la singularité. Même si l'auteur ne précise pas la fréquence des discussions à partir de laquelle un outil devient réellement interactif, ses études de cas (1995, p. 96, p. 100) indiquent que les systèmes de contrôle interactif font l'objet de réunions hebdomadaires ou mensuelles.

Les développements précédents montrent que les leviers de contrôle diagnostique et interactif semblent de prime abord incompatibles. Pourtant plusieurs auteurs (Tuomela 2005, Widener 2007, Sponem 2009, Naro et Travaillé 2010, Essid et Berland 2011...) s'inscrivent en faux en considérant que ces deux leviers de contrôle sont indissociables. En effet, ils remettent en cause "l'hypothèse d'indépendance relative de ces deux leviers de contrôle telle que la présente Simons » (Naro et Travaillé 2010). Ainsi, au lieu d'étudier ces leviers de contrôle de façon séparée, ces auteurs suggèrent d'explorer leurs interactions et de montrer leurs complémentarités. Selon Widener, la combinaison des deux leviers de contrôle aurait un impact positif sur la performance. De même, Sponem (2009, p. 567-571) considère qu' «il est nécessaire de combiner contrôles interactif et diagnostique pour permettre à l'entreprise de fonctionner; [le premier] favorisant l'apprentissage du fait de la capacité d'attention limitée des managers, [et permet de] voir comment le futur peut être différent du passé ; [et le second] permettant de s'assurer que les routines continuent à être accomplies et que l'organisation produit et atteint ses objectifs ».

Pour Naro et Travaillé, une réponse structurelle au paradoxe exploration/exploitation (au sens de March) réside dans l'implantation d'organisations «ambidextres» (O'Reilly et Tuchman 2004). Le 
contrôle interactif favoriserait l'exploration, qui est l'acquisition de nouveaux savoirs, tandis que le contrôle diagnostique permettrait l'exploitation des savoirs existants, c'est-à-dire leur utilisation et perfectionnement. Ainsi, «loin de s'opposer, [ces leviers de contrôle] constituent en fait les deux faces complémentaires d'un système de contrôle de gestion. En les conjuguant ainsi, l'organisation pourrait concilier deux exigences contradictoires : l'aptitude à innover, gérer les incertitudes stratégiques, saisir de nouvelles opportunités et favoriser l'éclosion des stratégies émergentes, au moyen d'un contrôle interactif; la maîtrise des variables critiques de performance, sur une sphère stable d'activités parvenues à maturité et dans le cadre de la mise en ceuvre des stratégies délibérées, à l'aide de systèmes de contrôle diagnostique » (Naro et Travaillé 2010, p. 61).

C'est dans ce contexte que se pose la question de l'articulation des deux leviers de contrôle : comment articuler le contrôle interactif et le contrôle diagnostique pour permettre d'une part le déploiement de la stratégie délibérée et d'autre part l'émergence de nouvelles stratégies ? Cette question est notamment pertinente dans le cas où un seul système de contrôle de gestion est utilisé pour répondre à ce double objectif. L'une des pistes évoquées concerne le cycle d'utilisation des systèmes de contrôle de gestion : "un même dispositif de contrôle ne pourrait-il pas être utilisé, selon différentes phases de son cycle de vie, tour à tour de manière interactive et de manière diagnostique?» (Naro et Travaillé 2010, p. 62). Les travaux empiriques de Tuomela et Essid et Berland semblent particulièrement intéressants pour obtenir de premiers éléments de réponses. Ces auteurs montrent que l'utilisation d'un système de contrôle de gestion peut évoluer dans le temps. On assiste alors à un «glissement » de l'utilisation interactive du système de contrôle de gestion vers une utilisation diagnostique et inversement. Chez Tuomela, le système de contrôle de gestion (appelé $3 K$ Scorecard) a d'abord été utilisé de manière diagnostique pour contrôler les facteurs clés de succès (FCS) de la stratégie, puis de manière interactive pour discuter des objectifs et des stratégies. Tandis que dans l'étude menée par Essid et Berland, le système de contrôle de gestion (appelé Energetix Way) initialement conçu dans une perspective interactive est devenu au fil du temps diagnostique pour pallier la surcharge cognitive: "la responsabilité sociale des entreprises, en multipliant les informations et donc en créant une surcharge cognitive, rend délicate une utilisation interactive des systèmes de contrôle, ainsi que l'implication des managers dans ces systèmes ». 
Bien que les travaux antérieurs soient clarificateurs sur l'évolution de l'utilisation d'un système de contrôle de gestion dans le temps, ils réduisent cependant la question de l'articulation au passage d'un levier de contrôle à l'autre durant le cycle de vie du système de contrôle de gestion. On peut alors s'interroger sur la nature de l'articulation des leviers de contrôle : cette dernière est-elle toujours «successive» ou peut-elle se manifester de façon «parallèle »? À notre connaissance, aucune étude empirique ne répond à ce questionnement qui, pourtant, semble essentiel dans la réflexion sur la conception des systèmes de contrôle de gestion dans les organisations. En effet, il s'agit de répondre à «l'un des dilemmes auquel doit faire face les concepteurs de systèmes de contrôle [de gestion] pour concilier exploration et exploitation» (Sponem 2009, p. 571). Cette recherche va donc s'intéresser à ce phénomène rarement étudié dans la littérature en montrant comment un système de contrôle de gestion peut être utilisé pour gérer simultanément des situations paradoxales. Autrement dit, la problématique de la recherche est d'étudier l'utilisation d'un seul et même système de contrôle de gestion à la fois comme un levier de contrôle diagnostique et comme un levier de contrôle interactif et, par conséquent, de comprendre l'articulation de ces deux modes d'usage.

\subsection{L'élaboration d'une grille d'analyse pour étudier l'objet de la recherche}

Comme nous venons de le voir, notre recherche s'inscrit dans la dialectique complémentaire des leviers de contrôle diagnostique et interactif. À la lumière de la revue de la littérature exposée précédemment, nous avons élaboré une grille d'analyse synthétisant les caractéristiques de ces deux leviers de contrôle (cf. tableau 1). Cette grille servira de référentiel pour étudier l'utilisation d'un système de contrôle de gestion en particulier : les indicateurs environnementaux d'une entreprise française du secteur des vins et spiritueux.

Finance-Contrôle-Stratégie, Vol. 16, N³, 2013 -Angèle RENAUD 

Tableau 1: Grille d'analyse des systèmes de contrôle
diagnostique/interactif

\begin{tabular}{|c|c|c|}
\hline Caractéristiques & Contrôle diagnostique & Contrôle interactif \\
\hline Objectifs & $\begin{array}{l}\text { Décliner la stratégie } \\
\text { délibérée en permettant } \\
\text { de fixer des objectifs, } \\
\text { de mesurer des } \\
\text { résultats et de corriger } \\
\text { les écarts }\end{array}$ & $\begin{array}{l}\text { Gérer les incertitudes } \\
\text { stratégiques, favoriser } \\
\text { l'apprentissage } \\
\text { organisationnel et } \\
\text { l'émergence } \\
\text { nouvelles stratégies }\end{array}$ \\
\hline Rôle des dirigeants & $\begin{array}{l}\text { Intervention limitée à } \\
\text { la fixation des objectifs } \\
\text { et à la résolution de } \\
\text { problèmes imprévus ou } \\
\text { complexes }\end{array}$ & $\begin{array}{l}\text { Implication personnelle } \\
\text { et fréquente à tous les } \\
\text { niveaux } \\
\text { l'organisation }\end{array}$ \\
\hline Rôle des fonctionnels & $\begin{array}{ll}\text { Rôle central dans la } \\
\text { préparation } & \text { et } \\
\text { l'interprétation } & \text { de } \\
\text { l'information: } \\
\text { construire et maintenir } \\
\text { le système, interpréter } \\
\text { les données, préparer } \\
\text { les rapports concernant } \\
\text { les exceptions, s'assurer } \\
\text { de l'intégrité et de la } \\
\text { fiabilité du système }\end{array}$ & $\begin{array}{l}\text { Rôle limité dans la } \\
\text { préparation } \\
\text { l'interprétation } \\
\text { résultats : collecter, } \\
\text { compiler les données, } \\
\text { faciliter le processus } \\
\text { interactif }\end{array}$ \\
\hline $\begin{array}{lr}\text { Processus } & \text { de } \\
\text { transmission } & \text { des } \\
\text { informations } & \end{array}$ & $\begin{array}{l}\text { Procédures formelles } \\
\text { de reporting }\end{array}$ & $\begin{array}{l}\text { Réunions hebdomadaire } \\
\text { ou mensuelle, débat et } \\
\text { dialogue fréquents }\end{array}$ \\
\hline $\begin{array}{l}\text { Fréquence d'interaction } \\
\text { entre les acteurs }\end{array}$ & $\begin{array}{l}\text { Ponctuelle, } \\
\text { exceptionnelle }\end{array}$ & Répétitive, continuelle \\
\hline $\begin{array}{l}\text { Indexation de la } \\
\text { rémunération } \\
\text { l'atteinte des objectifs }\end{array}$ & Forte & Faible \\
\hline $\begin{array}{l}\text { Glissement dans } \\
\text { l'utilisation avec le } \\
\text { temps (du diagnostique } \\
\text { vers l'interactif et } \\
\text { inversement) }\end{array}$ & Oui/non & Oui/non \\
\hline
\end{tabular}

Finance-Contrôle-Stratégie, Vol. 16, N³, 2013 -Angèle RENAUD 


\subsection{Les indicateurs environnementaux comme objet de recherche}

Avant de préciser les raisons qui nous conduisent à étudier les indicateurs environnementaux, il convient de définir cet objet de recherche. Les indicateurs environnementaux sont «des grandeurs, établies à partir de quantités observables ou calculables, reflétant de diverses façons possibles les impacts sur l'environnement occasionnés par une activité donnée ainsi que les efforts déployés pour les réduire, que ce soit sous forme de dépenses consenties en matière de gestion de l'environnement, de mise en place d'un système de management environnemental, d'obtention de certificats... Les quantités en question peuvent être aussi bien des quantités physiques de matières entrant dans un processus industriel (inputs, énergie, eau, matières premières...), ou provenant de ce processus, comme les produits de consommation ou intermédiaires, ou les rejets dans l'environnement sous forme de pollution atmosphérique, d'effluents liquides, de déchets solides toxiques, ou dangereux, ou inertes » (Tyteca 2002, Tyteca et al. 2002).

L'Organisation internationale de normalisation (ISO) propose de distinguer les indicateurs environnementaux en deux catégories : les indicateurs de performance environnementale (IPE) et indicateurs de condition environnementale (ICE) (Norme ISO 14031 2000). Les ICE sont considérés comme des indicateurs macro-économiques et, de ce fait, ne seront pas étudiés dans cette recherche. Nous allons focaliser notre réflexion sur les IPE. La norme ISO différencie deux groupes d'IPE : (1) les indicateurs de performance de management (IPM) qui produisent des informations sur les efforts accomplis par les dirigeants pour réduire les impacts environnementaux (ex. les ressources engagées dans les programmes environnementaux, le nombre d'audits environnementaux réalisés...) et (2) les indicateurs de performance opérationnelle (IPO) qui reflètent la performance environnementale des opérations de l'entreprise (ex. la quantité d'énergie utilisée par année ou par unité de produit, le nombre annuel de cas d'urgence, la quantité de

\footnotetext{
${ }^{3}$ Selon la norme ISO 14031 (2000), les ICE donnent des informations relatives à la condition locale, régionale, nationale ou mondiale de l'environnement. Leur élaboration incombe généralement aux agences gouvernementales, aux organisations non gouvernementales et aux instituts scientifiques et de recherche, plutôt qu'à des organisations individuelles, qui les utilisent dans le cadre de l'élaboration des normes et des réglementations environnementales, ou encore de la communication d'informations au public.
} 
déchets dangereux, recyclables ou réutilisables produits chaque année....).

Le choix des indicateurs environnementaux (IPE) comme objet d'étude se justifie par la dualité de leur représentation dans la littérature. Ces indicateurs sont utilisés tantôt de manière diagnostique et tantôt de manière interactive. En effet, selon la littérature (voir Janicot 2007, Rivière-Giordano 2007, Milne et Gray 2007, Henri et Journeault 2010, Damak-Ayadi 2010, Hopwood, Unerman et Fries 2010...) les IPE jouent deux rôles distincts dans les entreprises. D'un côté, ils sont regroupés dans un tableau de bord à usage interne pour piloter la performance environnementale et faciliter la prise de décision stratégique; et de l'autre côté, ils permettent d'effectuer un reporting environnemental pour s'assurer de la mise en œuvre de la stratégie de l'entreprise et pour "rendre compte auprès des différentes parties prenantes de ses activités et de leurs impacts sur la Société et l'environnement » (Damak-Ayadi 2010, p. 55). Ainsi, en étudiant l'utilisation des IPE dans l'entreprise «Maison de champagne » avec le cadre conceptuel des leviers de contrôle, nous verrons plus loin dans quelles mesures ce système de contrôle de gestion remplit ses deux rôles (pilotage interne et reporting externe) pour permettre à l'entreprise de fonctionner.

\section{La méthodologie de la recherche : une étude de cas dans le secteur des vins et spiritueux}

Pour répondre à la question de recherche, nous privilégions une méthodologie qualitative fondée sur une seule étude de cas. Après avoir justifié ce choix méthodologique (2.1), nous présentons le protocole de recueil et d'analyse des données (2.2) ainsi que le cas «Maison de champagne » dans une optique de contextualisation des données (2.3).

\subsection{La justification du choix de la méthode et du cas étudié}

Le choix de l'étude de cas comme stratégie de recherche découle de notre positionnement épistémologique et de l'objectif de la recherche. Nous nous inscrivons dans un «paradigme interprétativiste » dans la mesure où le but de notre étude est avant tout de comprendre et d'analyser un phénomène mal connu ou rarement exploré : l'articulation des usages diagnostique et interactif d'un seul et même système de contrôle de gestion. Pour ce faire, les acteurs 
(notamment les managers) sont placés au cœur de l'analyse afin de donner un sens au phénomène étudié. Dans ce type de paradigme dont la visée est essentiellement compréhensive, l'étude de cas est particulièrement adaptée, car elle permet «de saisir le sens subjectif et intersubjectif d'une activité humaine concrète à partir des perceptions et des actions des différents acteurs en présence. (...) le comportement humain et organisationnel ne peut se comprendre et s'expliquer qu'en relation avec les significations que les personnes donnent aux choses et à leurs actions » (Hlady Rispal 2002, p. 62). De plus, cette méthode est pertinente au regard de notre question de recherche. En effet, selon Yin (1989, p. 20), l'étude de cas est appropriée "lorsque se pose une question du type comment ou pourquoi à propos d'un ensemble contemporain d'événements sur lesquels le chercheur a peu ou pas de contrôle». En outre, notre choix méthodologique s'inscrit dans la lignée de nombreux chercheurs qui ont également mobilisé le cadre conceptuel de Simons pour examiner les systèmes de contrôle de gestion (voir Abernethy et Brownell 1999, Marginson 2002, Vaivio 2004, Tuomela 2005, Naro et Travaillé 2010, Essid et Berland 2011).

Pour mener à bien notre recherche, nous avons opté pour un cas unique permettant ainsi une investigation en profondeur du phénomène étudié et un effort de contextualisation des données, indispensable à la généralisation théorique des résultats de la recherche (Gavard-Perret et al. 2008). À ce propos, les travaux de March et al. (1991) montrent que l'unicité du cas ne remet pas en cause le potentiel de généralisation des résultats (Hlady Rispal 2002). Par ailleurs, le cas observé est à visée «instrumentale» (Stake 1994) puisque «son étude est polarisée par une question théorique générale» (David 2004). En effet, le questionnement fondamental de notre recherche porte davantage sur l'articulation des leviers de contrôle que sur le cas lui-même. Comme nous le verrons infra, le cas "Maison de champagne» présente «un intérêt fort mais [qui reste cependant] secondaire, car son rôle est de faciliter la compréhension de quelque chose d'autre, d'un intérêt externe» (Hlady Rispal 2002, p. 78), en l'occurrence les usages diagnostique et interactif d'un seul et même système de contrôle de gestion.

Au regard de notre question de recherche, deux raisons essentielles justifient le choix du cas «Maison de champagne». Premièrement, cette entreprise de vins et spiritueux a été sélectionnée, car elle appartient à un secteur d'activité caractérisé par un contexte écologique 
instable ${ }^{4}$. Cet environnement l'a amené à faire des choix stratégiques qui nécessitaient une remise en cause de ses pratiques viticoles pour mieux respecter l'environnement et répondre aux attentes de ses parties prenantes, tout en assurant sa rentabilité économique. Autrement dit, les activités de cette entreprise étant soumises à de fortes incertitudes stratégiques liées à des facteurs institutionnels (des législations et réglementations environnementales de plus en plus contraignantes notamment en matière d'utilisation des produits phytosanitaires), de marché (l'évolution des préférences des consommateurs vis-à-vis de la qualité environnementale des vins, des changements dans le comportement environnemental des concurrents) et de risques environnementaux (la difficulté à prévoir les risques d'accident, d'inondation, d'incendie ou encore l'épuisement des ressources naturelles comme l'eau indispensable à l'activité viticole...); il nous a semblé pertinent d'étudier la manière dont les dirigeants se servaient du contrôle de gestion pour gérer ces incertitudes stratégiques. En effet, à en croire Simons, ce type d'environnement (i.e. caractérisé par des incertitudes élevées) serait propice au contrôle interactif.

Deuxièmement, notre choix s'est porté sur cette entreprise, car les dirigeants ont formalisé leur système de contrôle de gestion pour traiter les incertitudes stratégiques en matière environnementale et répondre aux exigences de publication environnementale imposées par la loi NRE de 2001 (voir l'article 116 de la Loi n²001-420 du 15 mai 2001

4 En effet, la filière française des vins et spiritueux génère des impacts environnementaux considérables. Depuis les années 1960, l'utilisation des produits phytosanitaires s'est généralisée pour faciliter le travail des viticulteurs et réduire les coûts de production. On estime aujourd'hui, en France, à $20 \%$ la consommation des produits phytosanitaires pour des vignobles qui ne représentent que 3\% des terres agricoles. La France occupe ainsi le $3^{\text {ème }}$ rang mondial des consommateurs de produits phytosanitaires. Au début des années 2000, face à une conscience écologique du marché, le système législatif et réglementaire français est devenu plus contraignant, par exemple, en incitant fortement la filière à réduire sa consommation de désherbants et ses émissions de gaz à effet de serre liées aux activités de viticulture, de vinification et de conditionnement. C'est ainsi que, pour faire face à sa responsabilité environnementale, le Comité Champagne (CIVC) a réalisé en 2003 une analyse environnementale de toute la filière mettant ainsi en évidence quatre enjeux majeurs : la maîtrise des nuisances et des risques liés notamment à l'utilisation d'intrants (produits phytosanitaires, engrais chimiques de synthèse...), la préservation et la mise en valeur des terroirs, de la biodiversité et des paysages, la gestion responsable de l'eau, des effluents, des sous-produits et des déchets et le défi énergétique et climatique. (Pour en savoir plus, voir le site internet de l'Institut Français de la Vigne et du Vin : http://www.vignevin.com/publications/collectionitineraires.html). L'entreprise «Maison de champagne», qui fait l'objet de cette étude, s'inscrit dans cette dynamique. 
relative aux nouvelles régulations économiques et le décret d'application $n^{\circ}$ 2002-221 du 20 février 2002). Ce système de contrôle de gestion se présente ici sous la forme d'un ensemble d'indicateurs relevant de dimensions multiples de la performance environnementale. Dans notre étude, le caractère formel du système de contrôle de gestion est une condition sine qua non pour mobiliser les concepts de contrôle diagnostique et interactif. En effet, Simons (1994 p. 170) exclu explicitement le contrôle informel de son cadre conceptuel. En somme, cette entreprise réunissait les conditions nécessaires pour répondre à notre question de recherche.

\subsection{Le protocole de recueil et d'analyse des données}

L'étude de cas «Maison de champagne » a été réalisée sur une période de deux ans en combinant trois modes de recueil de données : l'entretien semi-directif, l'observation non participante et la collecte de documents écrits. Cette triangulation des méthodes permet de limiter le risque de subjectivité et de renforcer la «validité interne» de la recherche (Hlady Rispal 2002, Miles et Huberman 2003, Ayerbe et Missonier 2007).

Comme Simons (1991, p. 51-52), nos entretiens se sont focalisés sur les dirigeants, car c'est la manière dont ces derniers utilisent les systèmes de contrôle de gestion qui permet de distinguer les leviers de contrôle diagnostique et interactif. Ainsi, quatre entretiens semidirectifs ont été menés en profondeur auprès des principaux managers impliqués dans la démarche environnementale : le président-directeur général (PDG), le directeur qualité et environnement (QE) et le directeur vignes et vins. Ce dernier, en plus de la production, est également chargé de l'approvisionnement des raisins auprès de vignerons indépendants et de la communication des vins auprès des prescripteurs, des sommeliers et de la presse.

D'une durée moyenne de $1 \mathrm{~h} 30$, ces entretiens portaient sur la stratégie verte ${ }^{5}$ des dirigeants et sa déclinaison dans l'entreprise, les modes de gouvernance (les comités traitant des problématiques environnementales, les membres, la périodicité des réunions...), l'organisation de la fonction environnement, le fonctionnement du

\footnotetext{
${ }^{5}$ On entend par stratégie verte (environnementale ou écologique) un « ensemble de lignes directrices que les entreprises définissent afin de répondre à des pressions courantes internes et/ou externes et/ou d'anticiper l'évolution de l'environnement concurrentiel, des réglementations et de la demande » (Azzone et al. 1995).
} 
système de management environnemental (SME), les systèmes de contrôle de gestion utilisés pour piloter la performance environnementale et rendre des comptes aux parties prenantes, etc. En outre, les deux interviews accordées par le directeur QE ont été réalisées à un an d'intervalle, ceci dans le but de suivre l'évolution dans le temps de l'utilisation du système de pilotage et de reporting environnemental de l'entreprise.

Pour aller au-delà du discours des dirigeants, nous avons eu recours à l'observation non participante et à l'analyse documentaire. Cette observation consistait à prendre des notes lors des visites en entreprise sans pour autant s'impliquer dans son fonctionnement. En effet, contrairement à la recherche-intervention, notre objectif n'était pas d'introduire un changement dans l'organisation pour en observer les effets (Gavard-Perret et al.) mais plutôt de décrire et de comprendre des pratiques dans leur contexte. C'est ainsi que, par exemple, nous avons pu comparer les indicateurs présents dans le tableau de bord environnemental des dirigeants et ceux qui figuraient dans le rapport environnement de l'entreprise. En outre, pour vérifier et compléter nos données primaires, nous avons recueilli différents types de documents : la politique environnementale de l'entreprise, des procédures écrites, des comptes rendus de réunion, les rapports environnement des trois dernières années, des articles de presse, les pages environnementales du site internet de l'entreprise.

Après la collecte des données, nous avons effectué une analyse de contenu thématique. Cette analyse consistait à «repérer des noyaux de sens [des thèmes] qui composent la communication [i.e. le corpus des retranscriptions d'entretien et des documents] et dont la présence ou la fréquence d'apparition pourront signifier quelque chose pour l'objectif analytique choisi » (Bardin 2007, p. 137). Pour ce faire, nous avons réalisé une lecture «flottante » du corpus suivie essentiellement d'un «codage a priori » (Miles et Huberman 2003) en mobilisant la grille d'analyse élaborée à partir de la littérature (cf. supra tableau 1). Ce codage a été facilité par l'utilisation du logiciel d'analyse qualitative NVivo7. Compte tenu de la taille du corpus, nous nous limitons ici à un aperçu du codage effectué (cf. tableau 2). L'analyse proprement dite a été réalisée en recherchant les similarités et les différences par rapport au cadre conceptuel mobilisé au préalable. 
Tableau 2 : Extrait du codage

\begin{tabular}{|c|c|c|c|}
\hline Thème & \multicolumn{2}{|c|}{ Sous-thèmes } & Extraits codés \\
\hline \multirow[b]{2}{*}{$\begin{array}{c}\text { Rôles des } \\
\text { acteurs }\end{array}$} & Acteurs & \multicolumn{2}{|c|}{ Niveau d'implication des acteurs } \\
\hline & $\begin{array}{c}\text { Haut } \\
\text { dirigeant } \\
\text { (PDG) }\end{array}$ & $\begin{array}{c}\text { Implication } \\
\text { personnelle } \\
\text { et régulière } \\
\text { des } \\
\text { dirigeants } \\
\text { dans le } \\
\text { pilotage } \\
\text { environneme } \\
\text { ntal }\end{array}$ & $\begin{array}{l}\text { «Je pense que dans le domaine de } \\
\text { l'environnement l'exemple et le } \\
\text { leadership doivent venir } \\
\text { absolument du patron (...). On } \\
\text { fonctionne avec des comités de } \\
\text { pilotage et des comités } \\
\text { stratégiques, avec des réunions de } \\
\text { direction présidés par moi-même } \\
\text { (...). Et ensuite, dans tous les } \\
\text { projets d'innovation, présentés } \\
\text { dans un comité d'innovation que je } \\
\text { préside, il y a systématiquement } \\
\text { dans les critères de développement } \\
\text { des innovations un système } \\
\text { environnemental.» }\end{array}$ \\
\hline
\end{tabular}

\subsection{La présentation du cas «Maison de champagne»}

Créée au début du $18^{\mathrm{e}}$ siècle, «Maison de champagne » est une entreprise française du secteur des vins et spiritueux. Ayant opté pour une intégration verticale de la filière viticole, ses activités s'étendent de la culture de la vigne jusqu'à la distribution des bouteilles. Durant les vingt dernières années, cette ancienne entreprise familiale a connu une forte croissance économique notamment grâce aux investissements réalisés en France et à l'étranger (achat de domaines viticoles, exportation, etc.). Aujourd'hui, elle appartient à un Groupe multinational coté en bourse. Elle emploie 500 salariés sur une dizaine de sites d'exploitation.

Pour être compétitif sur un marché de plus en plus concurrentiel, l'entreprise s'est dotée d'une stratégie de «différenciation par le haut » basée sur des produits de qualité, innovants et respectueux de l'environnement naturel. Selon le directeur $\mathrm{QE}$, trois raisons essentielles expliquent la prise en compte des préoccupations écologiques dans la stratégie: d'abord, elle permet d'obtenir un avantage concurrentiel, de conquérir de nouveaux clients et d'attirer plus de fournisseurs sensibles à la protection de l'environnement (l'approvisionnement est une préoccupation majeure pour les dirigeants 
car seulement $20 \%$ des raisins sont produits par les vignobles de l'entreprise); ensuite, elle est source d'innovation par exemple en matière d'emballage et enfin, elle contribue à l'amélioration de l'image de l'entreprise.

Au début des années 1990, conscients de l'impact des questions écologiques sur la compétitivité et la rentabilité de l'entreprise, les dirigeants ont mis en place une série d'actions environnementales : la «viticulture raisonnée ${ }^{6}$, la réduction des consommations d'eau (notamment lors du nettoyage des pressoirs et des cuves, de l'irrigation des vignes et le pressurage des raisins), la maitrise des effluents (durant le processus de vinification et de distillation), la gestion des déchets (par la réduction des emballages à la source, le recyclage des cartons, plastiques, bois, etc.), la réduction des émissions de gaz à effet de serre (GES) issues des activités de transports (par exemple avec le choix du transport maritime ou ferroviaire au lieu du transport aérien...), l'écoconception (qui consiste à réduire les impacts environnementaux durant le cycle de vie du produit notamment grâce au choix des matériaux plus verts, la diminution du volume du produit ou de l'emballage, l'utilisation des produits recyclés dans la composition des emballages...). Cependant, ces différentes actions n'étaient pas toujours coordonnées, elles étaient parfois limitées à quelques sites opérationnels ou à des catégories d'acteurs (par ex. les vignerons ou les employés des ateliers de production) et les contrôles peu formalisés manquaient d'efficacité.

En 2001, l'arrivée d'un nouveau président-directeur général (PDG) marque un tournant décisif dans la gestion environnementale de l'entreprise. En effet, celui-ci s'implique personnellement dans la démarche, formalise la stratégie verte de l'entreprise et transforme le système de management en adoptant la norme internationale ISO 14001. Perçue comme une démarche globale et structurante, l'instauration du système de management environnemental (SME) a

\footnotetext{
${ }^{6}$ « La viticulture raisonnée est l'application à la viticulture du concept d'agriculture raisonnée. L'agriculture raisonnée correspond à des démarches globales de gestion d'exploitation qui visent, au-delà du respect de la réglementation, à renforcer les impacts positifs des pratiques agricoles sur l'environnement et à en réduire les effets négatifs, sans remettre en cause la rentabilité économique des exploitations. Au-delà des impératifs de la sécurité alimentaire des produits agricoles, qui s'imposent à toutes les productions, les modes de production raisonnée peuvent faciliter la maîtrise des risques sanitaires et contribuer à l'amélioration du bien-être animal. Ils permettent également de contribuer à l'amélioration des conditions de travail » (Source: IFV, Institut Français de la Vigne et du Vin).
} 
permis de fédérer l'ensemble du personnel et a favorisé «la reconnaissance des actions écologiques de l'entreprise auprès des parties prenantes, notamment des clients, des citoyens ou des pouvoirs publics »(Boiral 2006). Cette implantation a également entrainé des changements organisationnels comme la création d'une fonction environnement, composée d'un directeur qualité environnement (QE) directement rattaché au président et des responsables environnement dont la mission est de décliner la politique environnementale sur les sites opérationnels et dans les services. De plus, parallèlement aux systèmes de «contrôle de gestion traditionnel», de nouveaux dispositifs de contrôle (bilan carbone, budgets verts, plans d'action, indicateurs environnementaux...) ont été mis en place pour déployer la stratégie verte de l'entreprise en actions concrètes et mesurer les résultats.

Parmi ces systèmes de contrôle de gestion, notre intérêt s'est focalisé sur un ensemble d'indicateurs environnementaux qui sert à la fois d'outil de pilotage et de reporting dans cette entreprise. En mobilisant le cadre conceptuel des leviers de contrôle, nous analysons infra la manière dont les dirigeants utilisent ces indicateurs pour répondre à ce double objectif.

\section{L'analyse du système de pilotage et de reporting environnemental de la «Maison de champagne "}

Au sein de l'entreprise « Maison de champagne », les dirigeants se servent d'un seul et même système d'indicateurs environnementaux pour piloter la performance de l'entreprise autour des incertitudes stratégiques et pour rendre des comptes aux parties prenantes. Ces indicateurs mesurent différentes dimensions de la performance environnementale (cf. annexe 1). Selon l'objectif poursuivi, nous allons voir que ces indicateurs sont utilisés soit de manière interactive (3.1), soit de manière diagnostique (3.2). Pour finir, nous montrons comment ces deux usages s'articulent (3.3) pour permettre à l'entreprise de fonctionner.

\subsection{L'usage interactif des indicateurs environnementaux pour un pilotage stratégique}

Les indicateurs environnementaux sont utilisés pour éclairer les choix des dirigeants dans un contexte d'incertitude. Leur rôle est de 
focaliser l'attention organisationnelle sur les incertitudes stratégiques comme l'évolution du cadre réglementaire, des préférences des clients ou des initiatives des concurrents sur le plan environnemental, la disponibilité des ressources naturelles, la non-maîtrise des risques environnementaux... pour permettre le pilotage de l'entreprise vers la réalisation de ses objectifs. Lorsqu'ils servent d'instruments de pilotage, ces indicateurs sont personnellement et régulièrement suivis par les dirigeants. Ils sont regroupés dans un tableau de bord pour faciliter leur utilisation et favoriser la réflexion stratégique lors des différents comités de direction. De nouveaux objectifs et des plans d'action innovants émergent des débats suscités par ces indicateurs. En référence aux leviers de contrôle de Simons, cette utilisation des indicateurs peut être assimilée au contrôle interactif.

Nous détaillons ci-après les éléments qui caractérisent l'usage interactif de ces indicateurs.

Les dirigeants ont recours aux indicateurs environnementaux pour signaler les priorités stratégiques aux membres de l'entreprise et guider leur réflexion dans la recherche de nouvelles solutions. Par exemple, les dirigeants accordent une attention particulière aux indicateurs relatifs aux risques environnementaux (risques d'incendie, de pollution des eaux ou des sols par les produits phytosanitaires, risques sur la santé des viticulteurs et la biodiversité...). La maîtrise des risques est considérée comme un enjeu majeur, car une catastrophe écologique résultant d'une mauvaise gestion des risques pourrait remettre en cause les activités, voire la pérennité de l'entreprise :

«On est dans un système d'appellation d'origine contrôlée (AOC) où les mauvaises pratiques ont des conséquences directes sur nos activités. (...) On ne peut pas prendre le risque de se retrouver devant la scène avec un problème environnemental. On est sensible à un accident, à une contamination qui viendrait en quelques heures détruire toute l'image de la marque construite sur plusieurs années. (...) donc nous avons tout intérêt à inciter nos vignerons à pratiquer la viticulture raisonnée, limiter leur utilisation de produits phytosanitaires et intégrer le plus de bonnes pratiques viticoles dans leurs productions » (Directeur $\mathrm{QE})$.

Compte tenu de l'importance des problématiques environnementales pour l'entreprise «Maison de Champagne », les indicateurs font l'objet d'une surveillance au plus haut niveau de direction (par le PDG) et de la part des managers opérationnels à tous les niveaux de l'organisation : 
« Notre PDG est très impliqué, [il] est très vigilant sur le suivi des indicateurs et sur les actions qui sont menées au niveau environnemental, notamment dès que nous mettons en place un projet ou dès que nous rentrons dans la conception d'un projet. (...) Nous avons mis en place différents indicateurs au niveau des ateliers de production notamment au niveau des cuveries. On suit mois par mois l'évolution de la consommation d'eau (l'eau est beaucoup utilisée en terme de nettoyage); on suit également la consommation d'énergie qui est nécessaire au chauffage ou au refroidissement de nos cuveries. » (Directeur vignes et vins).

Les données environnementales sont interprétées et discutées lors de réunions en face-à-face entre le PDG et ses principaux collaborateurs (directeur $\mathrm{QE}$, directeur vignes et vins, directeur marketing, directeur juridique...). Elles sont traitées de manière transversale au cours des différents comités de direction «traditionnels». Puis, elles sont débattues de manière approfondie lors d'une réunion spécifique (la revue de direction) organisée par le comité de direction «environnemental »:

«On fonctionne avec des comités de pilotage et des comités stratégiques, avec des réunions de direction présidés par moi-même (...). Et ensuite, dans tous les projets d'innovation, présentés dans un comité d'innovation que je préside, il y a systématiquement dans les critères de développement des innovations un système environnemental. »(PDG).

«On a créé un comité de direction environnemental avec quelques membres du comité de direction, qui sont véritablement impliqués dans le système: directeurs de vignobles, directeur de production, directeur juridique, directeur marketing et le PDG. Les autres directeurs (directeur de la communication, directeur financier, directeurs commerciaux) ne font pas partie de la revue de direction, car ils ne sont pas concernés directement. Mais le jour où on a besoin d'eux, ils sont présents à la réunion.» (Directeur $\mathrm{QE}$ ).

Les données environnementales sont collectées quotidiennement mais la fréquence d'interactivité varie en fonction des comités, elle peut être mensuelle, trimestrielle ou annuelle. Globalement, cette fréquence peut être considérée comme élevée au regard des exigences de la norme ISO 14001. En effet, cette dernière ne recommande qu'une revue de direction annuelle pour traiter des problématiques environnementales :

"Les collectes de données se font chaque jour, et on a un bilan mensuel qui est sorti par l'équipe QSE (Qualité Sécurité 
Environnement) qui nous remonte ces indicateurs chaque fin de mois. Tous ces indicateurs sont regroupés dans un tableau de bord spécifique environnement. » (Directeur vignes et vins).

«Au niveau de notre TDB environnemental, on a un suivi mensuel (...). De façon annuelle, on a une vraie présentation avec un vrai engagement, suivi des résultats, de l'analyse des performances entre résultats et objectifs, et fixation des nouveaux objectifs. (...) Les comités innovations, c'est tous les mois; un certain nombre de bilans sont annuels. » (PDG).

«On a une revue de direction qui est annuelle. C'est une réunion spécifique à l'environnement. Ensuite, on a un bilan, enfin un point trimestriel, de tous les plans d'actions avec tous les directeurs. Et puis, on a un bilan mensuel avec l'ensemble des correspondants environnementaux. » (Directeur $\mathrm{QE})$.

En tant qu'objet fréquent de discussion, les indicateurs environnementaux favorisent l'apprentissage en structurant le dialogue entre les dirigeants et leurs collaborateurs autour du contenu détaillé, opérationnel et technique des impacts environnementaux des activités. Ces indicateurs donnent lieu à des débats qui stimulent l'innovation par la recherche de solutions alternatives moins néfastes pour l'environnement. Comme le mentionnait Vaivio (2004), les indicateurs non financiers sont propices aux apprentissages, car ils donnent lieu à des discussions sur l'interprétation des données et permettent ainsi d'externaliser des savoirs locaux.

«Il y a deux parties dans ces réunions: une partie opérationnelle qui permet de revoir les indicateurs (...); il y a aussi une partie plus stratégique où (...) les grands projets d'investissement sont systématiquement étudiés et cela permet de prendre en compte leurs impacts environnementaux. Par exemple, on a un projet qui s'appelle "X», c'est un projet d'investissement pour implanter de nouvelles lignes de production et des caves. Et donc on a décidé en revue de direction environnementale de mener ce projet en allant rechercher une certification $H Q E$ qui permet de réduire l'impact sur l'environnement à la fois au niveau de la réalisation des travaux mais aussi dans le fonctionnement $d u$ site une fois les investissements terminés. » (Directeur vignes et vins).

«Le système nous oblige, d'un point de vue stratégique, à nous orienter automatiquement vers des technologies de pointe. Aujourd'hui, dans nos choix des matériaux, on recherche ceux qui ont le moins d'impacts pour l'environnement, donc on doit consulter 
des experts, faire des recherches (...). C'est la même démarche pour les techniques viticoles, on va chercher des technologies qui permettent d'avoir des techniques plus douces pour l'environnement. » (Directeur QE).

Ce processus d'apprentissage aboutit à la définition de nouveaux objectifs et plans d'action innovants. Par exemple, les débats sur les risques phytosanitaires liés aux activités viticoles ont conduit les dirigeants à mettre en place des programmes de prévention des risques (tels que l'enherbement ou la «confusion sexuelle»). De même, la réflexion des dirigeants sur la réduction en amont (ou à la source) des impacts environnementaux liés aux produits a abouti à l'élaboration des programmes d'éco-conception des emballages :

«Pour obtenir des résultats, l'enherbement, à savoir la plantation d'herbe entre les vignes, est un élément clé car il permet de limiter d'une part l'utilisation de pesticides pour désherber et d'autre part la dispersion, par les eaux de ruissellement de ceux qui sont nécessairement utilisés pour protéger la vigne. Un itinéraire technique a été fixé pour adapter les mesures aux spécificités de chaque parcelle. Chaque année des avancées sont enregistrées. En 2005, 22\% de la surface du vignoble ont été enherbés, puis 44\% en 2006 et 55\% en 2007 (...) Initiative supplémentaire, [l'entreprise] favorise les alternatives possibles aux traitements chimiques. La confusion sexuelle est pratiquée. Au lieu de tuer les chenilles [qui abîment les grappes de raisins], il s'agit de saturer l'air de phéromones sexuelles en installant dans les vignes des capsules qui les émettent. Les mâles sont ainsi perturbés et n'arrivent plus à rencontrer les femelles, d'où absence d'accouplement, de fécondation, d'éclosion d'œuf et de naissance de chenille. Cette année, $78 \%$ du vignoble a été ainsi traité contre $20 \%$ pour l'ensemble du vignoble [de la région]. » (Rapport environnement).

«Notre objectif est de concevoir les nouveaux produits à partir de ces principes d'éco-conception. À ce jour, la prise en compte de l'environnement dans le développement de nos produits s'est traduite par deux optimisations principales : les emballages et le transport. [Au niveau des emballages], allègement des étuis $X$ ($25 \mathrm{~g}$ ) : gain de $7.5 \mathrm{t}$ de carton / an, diminution de $20 \%$ en poids des nouveaux habillages sur millésimes, utilisation de cartons comportant $61 \%$ de recyclé. » (Rapport environnement).

Les répercussions de ces nouvelles pratiques environnementales vont au-delà des activités de l'entreprise « Maison de champagne ». En effet, 
ces bonnes pratiques sont partagées avec les autres entités du Groupe et vont nourrir la réflexion stratégique de la Maison mère. Au sein du Groupe, une entité appelée « commission environnement » pilotée par la direction environnement est dédiée à l'analyse et au partage des retours d'expériences des filiales sur leurs actions environnementales. Ainsi, l'utilisation interactive des indicateurs au niveau de la filiale «Maison de Champagne » a contribué à l'amélioration de la stratégie verte du Groupe.

«La direction environnement, qui est directement rattachée au directeur général du Groupe, (...) réunit une fois par trimestre l'ensemble des correspondants environnement des Maisons [filiales] au sein d'une commission environnement dans le but de partager les meilleures pratiques de chacun. » (Rapport environnement).

Contrairement aux contrôleurs de gestion, dont les missions restent focalisées sur les dimensions économiques et financières de la performance, les membres de la fonction environnement sont très impliqués dans le reporting environnemental (nous y reviendrons plus loin). Le rôle de ces derniers reste cependant limité en matière de pilotage environnemental. En effet, ils facilitent le processus interactif en collectant les données environnementales et en organisant des réunions pour en débattre avec les managers opérationnels. Bien qu'ils contribuent à l'élaboration et l'interprétation des indicateurs, ils ne sont pas pour autant responsables des résultats. Ce sont les managers qui doivent s'assurer de l'atteinte des objectifs et prendre éventuellement des décisions correctives :

«On est beaucoup plus collectif et participatif. On décide ensemble des meilleurs moyens d'arriver aux objectifs sur l'année en cours. Ce n'est pas le service environnement qui va faire l'environnement à la place des autres. (...) Les directeurs ou chefs de services ont la responsabilité des impacts environnementaux de leurs services. Par exemple, le responsable de l'œnologie, qui a la responsabilité de toutes les cuveries, de la vinification, a également la responsabilité environnementale des impacts générés par ces cuveries. 》 (Directeur QE).

Les membres de l'entreprise sont peu évalués sur leurs performances environnementales. Des indicateurs environnementaux sont intégrés dans le calcul de l'intéressement, mais ils ne représentent au maximum que $15 \%$ de la prime globale. Il s'agit là d'une faible indexation des rémunérations sur les objectifs environnementaux, qui sont par ailleurs difficiles à déterminer car, outre les efforts du personnel, d'autres 
facteurs entrent en ligne de compte comme les aléas climatiques (ex. la consommation d'énergie des cuveries est difficile à prévoir avec certitude parce qu'elle varie selon que les vendanges sont précoces ou tardives). En outre, les dirigeants insistent davantage sur la visée incitative et sensibilisatrice de la mesure que sur l'évaluation des acteurs :

«On a mis des critères environnementaux dans l'intéressement des salariés (ex. des consommations d'eau, d'énergie, la qualité du tri sélectif. (...) en termes de pondération, c'est un peu moins important que les autres [critères classiques]. Ça reste quelque chose d'un peu symbolique, mais (...) c'est quand même une carotte.» (Directeur QE).

\subsection{L'usage diagnostique des indicateurs environnementaux pour rendre des comptes}

Pour des raisons d'image et de réglementation, l'entreprise «Maison de champagne » est amenée à publier régulièrement ses indicateurs environnementaux. En effet, cette entreprise appartient à un Groupe coté sur un marché réglementé français, qui est soumis à l'article 116 de la loi NRE. Donc à ce titre, elle doit rendre compte des conséquences environnementales de ses activités économiques. Pour effectuer ce reporting environnemental, nous avons constaté que le directeur QE transmettait les mêmes indicateurs environnementaux (i.e. ceux utilisés de manière interactive par les dirigeants de l'entreprise) aux instances de direction du Groupe (la direction environnement et la direction générale de la Maison mère). Outre ces parties prenantes internes, les indicateurs sont aussi communiqués aux parties prenantes externes (clients, fournisseurs, associations, collectivités territoriales, pouvoirs publics, société civile...) via le rapport environnement annuel et le site Internet de l'entreprise :

«On est un Groupe côté en Bourse (...). On fait tous les ans un bilan social et un bilan environnemental complets, qui sont publiés dans le rapport annuel du Groupe auquel nous appartenons. Cela fait partie de la communication financière. » (PDG).

En référence à Simons, plusieurs éléments montrent qu'il s'agit d'un usage diagnostique.

L'entreprise «Maison de champagne » étant une filiale intégrée, sa stratégie verte est définie au niveau de la banche "vins et spiritueux » du Groupe. Le reporting environnemental sert donc à vérifier l'atteinte 
des objectifs fixés par la Maison mère. Il permet aussi de rendre visible auprès des parties prenantes externes les efforts de l'entreprise dans le domaine environnemental. En effet pour des questions d'image, les bonnes performances environnementales sont mises en avant dans les rapports communiqués aux parties prenantes et les revues de presse disponibles sur le site Internet de l'entreprise. Pour crédibiliser sa démarche environnementale, le Groupe fait auditer son reporting par une tierce partie.

«La stratégie environnementale, on la dessine, on l'élabore avec les gens $d u$ Groupe, au dessus quoi. C'est une stratégie environnementale par branche d'activité. Il y a une tendance, ces derniers temps, à ce que dans chaque branche il y ait beaucoup de synergies mises en ouvre, même entre les différentes Maisons du Groupe. Mais chaque Maison garde son autonomie, enfin, sa façon de piloter les choses, même si les objectifs ou les indicateurs sont communs au niveau des performances. » (Directeur $\mathrm{QE}$ ).

«L reporting des indicateurs environnementaux permet de suivre la performance environnementale des Maisons et de communiquer des données environnementales à l'ensemble des parties prenantes du Groupe. Depuis 2003 il est certifié, comme les données financières, par les commissaires aux comptes du Groupe.» (Rapport environnement).

Les données environnementales de la filiale sont transmises à la direction environnement du Groupe selon une procédure formelle de reporting :

«L'ensemble des règles de consolidation et de calcul sont définies dans le protocole de reporting environnemental [du Groupe], mis à jour annuellement et tenu à disposition du public pour consultation par la direction environnement. (...) Le système utilisé pour le reporting environnemental est un outil interne de type excel-VBA. Deux fichiers sont envoyés dès l'automne à chacune des Maisons du Groupe : un fichier compilant des données corporate : formation, emballage, audits... un fichier compilant des données spécifiques aux sites industriels : consommations d'eau et d'énergie, production de déchets (quantité et types de déchets), traitement des déchets...» (Rapport environnement).

Le rôle du directeur QE et des spécialistes de l'environnement est central dans ce reporting. En effet, ils sont chargés de collecter, consolider et vérifier la fiabilité des données environnementales au niveau des différents sites de la filiale. Leur rôle peut être assimilé aux 
missions traditionnellement dévolues aux contrôleurs de gestion dans le domaine financier. Malgré les similitudes avec le processus de reporting financier, ces derniers sont absents du processus de reporting environnemental. Cette fonction est assumée par les fonctionnels de l'environnement.

«On a de multiples activités dans notre entreprise et on a plusieurs sites. Donc, pour la même problématique, on est amené à voir des populations complètement différentes de personnes, aussi bien des vignerons que des marketings, on passe d'un extrême à l'autre. (...) Pour les indicateurs environnementaux (consommations d'eau, d'énergie, etc.), les données sont collectées et, mensuellement, nous faisons un cumul. On gère l'ensemble, mais on essaye autant que faire se peut de les avoir par secteur d'activité, puisqu'on est dans la philosophie d'implication, de responsabilisation de chacun. » (Directeur QE).

\subsection{L'articulation des leviers de contrôle diagnostique et interactif}

Nous venons de voir qu'un seul et même système d'indicateurs environnementaux est utilisé de deux manières distinctes dans l'entreprise «Maison de champagne ». Il permet tantôt de déployer la stratégie verte du Groupe au sein de l'entreprise en veillant à la réalisation des objectifs fixés par la Maison mère et tantôt de favoriser l'émergence de nouvelles initiatives stratégiques en créant des débats réguliers à différents niveaux de l'organisation autour des données environnementales. Autrement dit, en référence au cadre conceptuel de Simons, ce système d'indicateurs sert à la fois de levier de contrôle diagnostique et de levier de contrôle interactif. Le tableau 3 résume les caractéristiques diagnostique et interactive de ce système. 
Tableau 3: Grille d'analyse contrôle diagnostique/interactif appliquée au système d'indicateurs environnementaux

\begin{tabular}{|c|c|c|}
\hline Caractéristiques & Contrôle diagnostique & Contrôle interactif \\
\hline Objectifs & $\begin{array}{l}\text { Décliner la stratégie } \\
\text { définie par la Maison } \\
\text { mère au niveau de } \\
\text { l'entreprise (filiale) en } \\
\text { vérifiant l'atteinte des } \\
\text { objectifs }\end{array}$ & $\begin{array}{l}\text { Gérer les risques } \\
\text { environnementaux, } \\
\text { favoriser l'apprentissage } \\
\text { organisationnel pour } \\
\text { proposer de nouveaux } \\
\text { objectifs ou des plans } \\
\text { d'actions innovants, } \\
\text { voire pour influencer la } \\
\text { stratégie initiale du } \\
\text { Groupe }\end{array}$ \\
\hline Rôle des dirigeants & $\begin{array}{l}\text { Intervention limitée de } \\
\text { la Maison mère à la } \\
\text { fixation des objectifs et } \\
\text { à la résolution de } \\
\text { problèmes imprévus ou } \\
\text { complexes }\end{array}$ & $\begin{array}{l}\text { Implication personnelle } \\
\text { et fréquente du PDG de } \\
\text { la filiale et des managers } \\
\text { opérationnels à tous les } \\
\text { niveaux de l'organisation }\end{array}$ \\
\hline $\begin{array}{l}\text { Rôle des fonctionnels } \\
\text { (membres de la } \\
\text { fonction } \\
\text { environnement) }\end{array}$ & $\begin{array}{l}\text { Rôle central dans le } \\
\text { reporting } \\
\text { environnemental }\end{array}$ & $\begin{array}{l}\text { Rôle limité dans le } \\
\text { pilotage environnemental }\end{array}$ \\
\hline $\begin{array}{lr}\text { Processus } & \text { de } \\
\text { transmission } & \text { des } \\
\text { informations } & \end{array}$ & $\begin{array}{l}\text { Procédures formelles } \\
\text { de reporting (règles de } \\
\text { calcul et de } \\
\text { consolidation définies } \\
\text { au niveau du Groupe) }\end{array}$ & $\begin{array}{l}\text { Réunions mensuelles, } \\
\text { trimestrielles } \\
\text { annuelles : comités } \\
\text { stratégique, de pilotage, } \\
\text { d'innovation, RDD, etc. }\end{array}$ \\
\hline $\begin{array}{l}\text { Fréquence } \\
\text { d'interaction entre les } \\
\text { acteurs }\end{array}$ & $\begin{array}{l}\text { Ponctuelle, } \\
\text { exceptionnelle }\end{array}$ & Répétitive, continuelle \\
\hline $\begin{array}{lr}\text { Indexation de la } \\
\text { rémunération } & \text { sur } \\
\text { l'atteinte des objectifs } \\
\text { environnementaux }\end{array}$ & Forte & $\begin{array}{l}\text { Faible : les indicateurs } \\
\text { environnementaux } \\
\text { représentent moins de } \\
15 \% \text { de l'intéressement }\end{array}$ \\
\hline $\begin{array}{l}\text { Glissement dans } \\
\text { l'utilisation avec le } \\
\text { temps (du diagnostique } \\
\text { vers l'interactif et } \\
\text { inversement) }\end{array}$ & Ouitnon & Ouitnon \\
\hline
\end{tabular}

Finance-Contrôle-Stratégie, Vol. 16, N³, 2013 -Angèle RENAUD 
Cependant, toutes les caractéristiques des leviers de contrôle évoquées dans la littérature n'ont pas été relevées dans le fonctionnement du système d'indicateurs environnementaux. En effet, contrairement aux travaux de Tuomela et d'Essid et Berland qui montrent un «glissement » des systèmes de contrôle de gestion dans le temps, d'un usage diagnostique vers un usage interactif (et inversement), notre étude met en évidence une utilisation «simultanée » d'un seul et même système de contrôle de gestion. Autrement dit, le système d'indicateurs environnementaux est à la fois diagnostique et interactif, il n'évolue pas d'un levier de contrôle à l'autre. Son « ambidextérité » permet à l'entreprise de fonctionner aussi bien dans la déclinaison de sa stratégie verte que dans l'émergence de nouvelles initiatives stratégiques.

Dans ce contexte, l'articulation diagnostique et interactive du système de contrôle de gestion se fait en «parallèle » (cf. tableau 4) : (1) d'un côté, le système de contrôle de gestion est utilisé de manière diagnostique pour remonter les données au niveau de la Maison mère afin de prouver la mise en œuvre effective de la stratégie verte définie au niveau du Groupe et rendre compte aux parties prenantes externes; (2) et de l'autre côté, au sein de la filiale «Maison de champagne », le système de contrôle de gestion est utilisé de manière interactive pour piloter la performance environnementale locale et tenter d'améliorer les objectifs, plans d'actions et la stratégie verte définis initialement.

Tableau 4 : Articulation «en parallèle » des usages diagnostique et interactif du système d'indicateurs environnementaux

\begin{tabular}{|l|l|l|}
\hline $\begin{array}{l}\text { Rôles du système de Types de contrôle } \\
\text { contrôle de gestion }\end{array}$ & $\begin{array}{c}\text { Contrôle } \\
\text { diagnostique }\end{array}$ & $\begin{array}{c}\text { Contrôle } \\
\text { interactif }\end{array}$ \\
\hline $\begin{array}{l}\text { Reporting environnemental vis-à-vis des parties } \\
\text { prenantes internes (direction environnement et } \\
\text { direction générale du Groupe) et externes } \\
\text { (clients, fournisseurs, citoyens, pouvoirs publics } \\
\text { et autorités locales, ..) }\end{array}$ & (1) & \\
\hline $\begin{array}{l}\text { Contrôle interne de la performance } \\
\text { environnementale (au niveau de la filiale) }\end{array}$ & & (2) \\
\hline $\begin{array}{l}\text { Émergence de nouveaux objectifs et plans } \\
\text { d'actions innovants au niveau de la filiale, } \\
\text { alimentation de la réflexion stratégique du } \\
\text { Groupe grâce aux retours d'expérience }\end{array}$ & & (2) \\
\hline
\end{tabular}


Il semble que l'artisan de cette articulation soit le directeur qualité environnement (QE). En effet, ce dernier conjugue ses missions de reporting imposées par la direction du Groupe et son rôle de conseiller auprès des dirigeants de la filiale. Il veille à la collecte des données environnementales, leur compilation et leur remontée à la direction environnement du Groupe, qui à son tour les consolide avec les données d'autres entités pour alimenter la réflexion stratégique de la Maison mère et pour élaborer le rapport environnement annuel du Groupe. Parallèlement à ses missions de reporting, le directeur $\mathrm{QE}$ accompagne les dirigeants de la filiale dans l'exploration de nouvelles solutions environnementales notamment lors des différents comités de direction (stratégique, pilotage, innovation, RDD...). Il permet ainsi de trouver un équilibre entre les usages diagnostique et interactif du système de contrôle de gestion.

\section{Discussion et conclusion}

L'objet de la recherche était de comprendre l'articulation des usages diagnostique et interactif d'un seul et même système de contrôle de gestion. Pour cela, nous avons mobilisé le cadre conceptuel des leviers de contrôle de Simons et des travaux empiriques qui en découlent (Tuomela, Naro et Travaillé, Essid et Berland) afin d'étudier le fonctionnement d'un système d'indicateurs environnementaux dans une entreprise française du secteur des vins et spiritueux sujette à de fortes incertitudes stratégiques dans le domaine environnemental. Les résultats du cas «Maison de champagne» suggèrent qu'un seul et même système de contrôle de gestion peut être utilisé pour gérer simultanément deux situations paradoxales: le déploiement d'une stratégie délibérée et l'émergence d'une nouvelle stratégie. Plusieurs enseignements théoriques, managériaux et méthodologiques peuvent être tirés de ce cas.

Sur le plan théorique, cette recherche tend à répondre au dilemme de l'exploitation versus exploration des savoirs (March). Selon Simons, la réponse à ce dilemme se trouve dans une «division du travail» entre contrôle diagnostique et contrôle interactif (Naro et Travaillé, Essid et Berland), l'un favorisant l'exploitation et l'autre l'exploration. Pour l'auteur, si une entreprise dispose de (n) systèmes de contrôle de gestion, (n-1) de ces systèmes seraient utilisés de manière diagnostique, tandis qu'un seul servirait à un usage interactif. En mettant en évidence la capacité d'un seul et même système de contrôle de gestion à 
contribuer de manière simultanée à ces deux types d'apprentissage, nos résultats viennent nuancer les propos de l'auteur. En effet dans cette étude, nous avons montré qu'un seul et même système d'indicateurs environnementaux est utilisé à la fois de manière diagnostique pour surveiller l'atteinte des objectifs fixés par la Maison mère et de manière interactive pour fournir un signal en faveur de la recherche de nouveaux savoirs permettant d'améliorer la stratégie de l'entreprise. Dans ce contexte, les dirigeants ne se contentent plus de la routine organisationnelle, de constater et corriger les écarts entre les objectifs stratégiques et les performances environnementales réalisées; ils cherchent à innover en stimulant le dialogue autour des incertitudes stratégiques et en débattant avec leurs subordonnés pour rechercher des solutions alternatives favorables à l'environnement et rentables pour l'entreprise. Ainsi, nous proposons que les recherches futures en contrôle de gestion explorent, parmi les (n) systèmes de contrôle de gestion dont dispose l'entreprise, la manière dont un seul et même système associe la routine à l'innovation.

Bien que cette recherche s'inscrive dans la lignée des travaux de Tuomela, Naro et Travaillé, Essid et Berland, qui apportent un éclairage certain sur les interactions entre les leviers de contrôle, elle se différencie néanmoins de ces derniers en montrant que l'articulation des usages diagnostique et interactif d'un système de contrôle de gestion peut se faire « en parallèle » et pas systématiquement « en glissement». En effet, dans ces travaux antérieurs, l'accent est mis sur l'évolution des systèmes de contrôle de gestion durant leur cycle de vie, ces derniers passant du levier diagnostique au levier interactif et inversement. Dans cette optique, le contrôle de gestion est étudié de façon linéaire par rapport au temps : on distingue alors les phases avant, pendant et après l'action. Mais le contrôle de gestion recouvre une réalité complexe qui ne peut être réduite à une succession de phases. Les futures études devraient davantage explorer la dynamique de ce processus plutôt que sa linéarité.

Sur le plan managérial, cette recherche nous invite à nous interroger sur l'intérêt d'utiliser un système de contrôle de gestion «ambidextre » pour répondre à des objectifs divergents. L'utilisation de ce système permet-il de réduire le nombre de systèmes de contrôle de gestion dans les entreprises et de ce fait d'éviter la dispersion de l'attention des dirigeants ? L'implantation d'un tel système conduit-elle les entreprises à être plus performantes ? Quels pourraient être les effets pervers d'un tel dispositif? Cette recherche pose également la question 
du rôle des contrôleurs de gestion dans le processus de contrôle de gestion environnemental. Ceux-ci étant absents du processus, leurs missions sont actuellement dévolues aux fonctionnels de l'environnement qui apparaissent désormais comme leurs nouveaux «concurrents». C'est donc à juste titre que Bouquin (2004, p. 94) affirme : «à mesure que certains contrôleurs se sont coupés du réel en se repliant sur l'unique logique comptable et budgétaire, ils se sont trouvés contournés par d'autres fonctionnels poursuivant, plus près des opérateurs, les mêmes buts ». De plus dans notre étude, les fonctionnels de l'environnement se retrouvent au cœur de la problématique d' "ambidextérité », car ils veillent à la mise en œuvre de la stratégie définie par le Groupe et accompagnent les dirigeants de l'entreprise (la filiale) dans la recherche de nouvelles initiatives stratégiques. Ils sont donc directement confrontés au dilemme de l'exploitation et de l'exploration des savoirs.

Sur le plan méthodologique, comme la plupart des recherches en contrôle de gestion, cette étude est essentiellement basée sur le discours des acteurs interrogés, la collecte de documents et l'observation non participante. Selon Gavard-Perret et al., ce dispositif méthodologique a pour avantage de limiter des biais de comportement des acteurs observés (les personnes qui se savent observées modifient leur comportement dans le sens d'un ajustement aux attentes supposées de l'observateur) et de garder une distance objective nécessaire à la remontée théorique. Cependant, ce dispositif ne permet pas d'accéder véritablement aux pratiques de contrôle mais simplement au discours sur les pratiques. En effet, «l'étude des pratiques n'est possible que dans le cadre d'une présence prolongée dans l'entreprise, avec un niveau d'intégration élevé du chercheur à l'organisation, pour lui permettre de partager le quotidien de l'entreprise et de saisir les moments exceptionnels et imprévisibles où se jouent des écarts, des surprises et des inflexions »(Lorino, 2008, p. 165). Ainsi, la recherche actuelle pourrait être améliorée en allant plus loin dans la description des mécanismes de l'articulation des usages diagnostique et interactif d'un système de contrôle de gestion, il conviendrait alors d'adopter des méthodes d'observations ethnographiques: «des observations journalières, notées dans un journal de bord, des entretiens non directifs enregistrés et retranscrits, et la collecte de copies de dossiers et de rapports internes confidentiels » (Gavard-Perret et al. 2008, p. 156). Par ailleurs, outre le contexte environnemental, la recherche gagnerait à être répliquée dans les autres domaines de la RSE pour voir 
dans quelles mesures les résultats obtenus sont transférables. Par exemple, il serait intéressant d'étudier l'intégration des différentes dimensions de la RSE dans un seul et même système de contrôle de gestion, à l'instar du Sustainability Balanced Scorecard, et voir s'il peut faire l'objet d'un double usage diagnostique et interactif.

\section{Bibliographie}

Abernethy M.A. et Brownell P. (1999), « The Role of Budgets in Organizations Facing Strategic Change : an Explorary Study », Accounting, Organizations and Society, vol. 24 n 3, p. 189-204.

Anthony R.N. (1988), Management Control Function, Harvard Business School Press.

Ayerbe C. et Missonier A. (2007), «Validité interne et validité externe de l'étude de cas : Principes et mise en oeuvre pour un renforcement mutuel », Finance Contrôle Stratégie, vol. 10, n 2, p. 37-62.

Azzone G., Bianchi R., Mauri R. et Noci G. (1997), « Defining Operating Environmental Strategies : Programmes and Plans within Italian Industries », Environmental Management and Health, vol. 8, $\mathrm{n}^{\circ}$ 1, p. 4-19.

Bardin L. (2007), L'analyse de contenu (éd. 1ère), Presse Universitaire de France.

Bisbe J. et Otley D. (2004), "The Effects of the Interactive Use of Management ", Accounting, Organizations and Society, vol. 29, p. 709-737.

Boiral O. (2006), «L'environnement en management et le management environnemental : enjeux et perspectives d'avenir », in O. Aktouf, O. Boiral, E. Mehran et A.L. Saives (éds.), Le management entre tradition et renouvellement (éd. 4e), Gaëtan Morin éditeur.

Bouquin H. (2004), Le contrôle de gestion (éd. 6e), Presses Universitaires de France.

Damak-Ayadi S. (2010), « Le reporting social et environnemental suite à l'application de la loi NRE en France », Comptabilité, Contrôle, Audit, tome 16, vol. 1, p. 53-82.

David A. (2004), «Études de cas et généralisation scientifique en sciences de gestion », $13^{\text {ème }}$ Conférence de l'Association Internationale de Management Stratégique, Le Havre. 
Essid M. et Berland N. (2011), «Les impacts de la RSE sur les systèmes de contrôle », Comptabilité, Contrôle, Audit, tome 17, vol. 2, p. $59-88$.

Gavard-Perret M.L., Gotteland D, Haon C. et Jolibert A. (2008), Méthodologie de la recherche : réussir son mémoire ou sa thèse en sciences de gestion, Pearson Education France.

Henri J.F. et Journeault M. (2010), « Eco-control : the Influence of Management Control System on Environmental And Organizational Performance », Accounting, Organizations and Society, vol. 35, $\mathrm{n}^{\circ} 1$, p. 63-80.

Hlady Rispal M. (2002), La méthode des cas. Applications à la recherche en gestion, De Boeck Université.

Hopwood A., Unerman J. et Fries J. (2010), Accounting for Sustainability Practical Insights, Earthscan.

Janicot L. (2007), « Les systèmes d'indicateurs de performance environnementale (IPE), entre communication et contrôle », Comptabilité Contrôle Audit, tome 13, vol. 1, p. 47-68.

Lorino, P. (2008), « Méthodes de recherche en contrôle de gestion : une approche critique », Finance Contrôle Stratégie, vol. 11, numéro hors serie, p. 149-175.

March J.G. (1991), « Exploration and Exploitation in Organizational Learning », Organization Science, vol. 2, n 1, p 71-87.

Marginson D.E. (2002), « Management Control Systems and their Effects on Strategy Formation at Middle-Management Levels : Evidence from a U.K. Organization ». Strategic Management Journal, vol. 23, n 11, p. 1019-1031.

Miles M. et Huberman M. (2003), Analyse des données qualitatives, De Boeck.

Milne M. et Gray R. (2007), « Future Prospects for Corporate Sustainability Reporting », in J. Unerman, J. Bebbington et B. O'Dwyer, Sustainability Accounting and Accountability, p. 184-207, Routledge.

Naro G. et Travaillé D. (2010), «Construire les stratégies avec le Balanced Scorecard : vers une approche interactive du modèle de Kaplan et Norton », Finance Contrôle Stratégie, vol. 13, n 2, p. 3366.

Norme ISO 14031 (2000), Management environnemental - Évaluation de la performance environnementale - Lignes directrices, AFNOR.

O'Reilly C.A. et Tushman M.L. (2004), Ambidextrous Organization, Harvard Business Review, vol. 10, n 4, 74-81.

Finance-Contrôle-Stratégie, Vol. 16, N³, 2013 -Angèle RENAUD 
Rivière-Giordano G. (2007), " Comment crédibiliser le reporting sociétal ? », Comptabilité Contrôle Audit, tome 13, vol. 2, p. 127-148. Simons R. (1987), "Planning, Control and Uncertainty: a Process View », in X.J. Bruns, R.S. Kaplan (éds), Accounting and Management: Field Study Perspectives, Harvard Business School Press.

Simons R. (1990), "The Role of Management Control Systems in Creating Competitive Advantage : New Perspectives », Accounting, Organizations and Society, vol. 15, n ${ }^{\circ} 1-2$, p. 127-143.

Simons R. (1991). "Strategic Orientation and Top Management Attention to Control Systems », Strategic Management Journal, vol. 12, p. 49-62.

Simons R. (1994), "How New Managers Use Control Systems as Levers of Strategic Renewal », Strategic Management Journal, vol. $15, n^{\circ} 3$, p. 69-189.

Simons R. (1995), Levers of Control : How Managers Use Innovative Control Systems To Drive Strategic Renewal, Harvard Business School Press.

Simons R. (2000), Performance Measurement and Control Systems For Implementing Strategy, Prentice Hall.

Sponem S. (2009), Contrôle et apprentissage organisationnel, in B. Colasse, Encyclopédie de Comptabilité, Contrôle de gestion et Audit, Economica, p. 563-574.

Stake R.E. (1994), « Case Studies », in N.K. Denzin et Y.S. Lincoln (éds.), Strategies of Qualitative Inquiry, Sage Publications, vol. 2, chap. 4, p. 86-109.

Tuomela T.S. (2005), « The Interplay of Different Levers of Control : a Case Study of Introducing a New Performance Measurement System », Management Accounting Research, vol. 16, n 3, p. 293-320.

Tyteca D. (2002), « Problématique des indicateurs environnementaux et de développement durable », Congrès de la SIM (Société de l'Industrie Minérale), octobre, Liège.

Tyteca D., Carlens J., Berkhout F., Hertin J., Wehrmeyer W. et Wagner M. (2002), « Corporate Environmental Performance Evaluation : Evidence from the MEPI Project », Business Strategy and the Environment 11 (1), p. 1-13.

Vaivio J. (2004), « Mobilizing Local Knowledge with "Provocative" non-Financial Measures », European Accounting Review, vol. 13, n 1 , p. 39-71. 
Widener S.K. (2007), "An Empirical Analysis of The Levers of Control Framework », Accounting, Organizations and Society, vol. 32, $\mathrm{n}^{\circ}$ 7-8, p. 757-788.

Yin R.K. (1989), Case Study Research : Design and Methods (éd. 2e), Applied Social Research Methods Series, vol. 5, Sage Publications.

\section{Annexe 1: Le système d'indicateurs environnementaux de «Maison de champagne »}

\begin{tabular}{|c|c|c|c|c|}
\hline $\begin{array}{c}\text { Types } \\
\text { d'indicateurs }\end{array}$ & $\begin{array}{c}\text { Dimensions } \\
\text { mesurées } \\
\text { de la performance }\end{array}$ & Indicateurs & Objectif & $\begin{array}{l}\text { Progression } \\
\text { avec } \\
\text { échéancier }\end{array}$ \\
\hline \multirow{5}{*}{ IPM } & $\begin{array}{l}\begin{array}{l}\text { Pratique de la } \\
\text { viticulture }\end{array} \\
\text { raisonnée pour } \\
\text { limiter les risques }\end{array}$ & $\begin{array}{l}\text { Surface du vignoble exploité } \\
\text { selon la viticulture raisonnée } \\
\text { (en \%) }\end{array}$ & & \\
\hline & $\begin{array}{l}\text { Maitrise des risques } \\
\text { environnementaux }\end{array}$ & $\begin{array}{l}\text { Nombre de sites certifiés } \\
\text { ISO } 14001 \\
\text { Nombre et résultats des } \\
\text { audits réalisés (audits } \\
\text { internes, audits externes) } \\
\text { Formation des auditeurs } \\
\text { internes et du personnel (en } \\
\text { heures) }\end{array}$ & & \\
\hline & $\begin{array}{lcl}\text { Respect de la } \\
\text { réglementation }\end{array}$ & $\begin{array}{l}\text { Degré de conformité aux } \\
\text { réglementations (en \%) }\end{array}$ & & \\
\hline & $\begin{array}{l}\text { Lien entre la } \\
\text { performance } \\
\text { environnementale } \\
\text { et la performance } \\
\text { financière }\end{array}$ & $\begin{array}{l}\text { Budget des dépenses et } \\
\text { investissements pour } \\
\text { prévenir les impacts de } \\
\text { l'activité } \\
\text { l'environnement (en } \\
\text { euros): protection de l'air, } \\
\text { gestion des eaux usées, des } \\
\text { déchets, protection de la } \\
\text { biodiversité, recherche et } \\
\text { développement... }\end{array}$ & & \\
\hline & $\begin{array}{l}\text { Implication des } \\
\text { parties prenantes }\end{array}$ & $\begin{array}{l}\text { Nombre de visites par site } \\
\text { du grand public } \\
\text { Développement de } \\
\text { partenariat avec les autorités } \\
\text { et les collectivités locales } \\
\text { Partage des bonnes pratiques } \\
\text { avec les vignerons et } \\
\text { l'interprofession }\end{array}$ & & \\
\hline
\end{tabular}



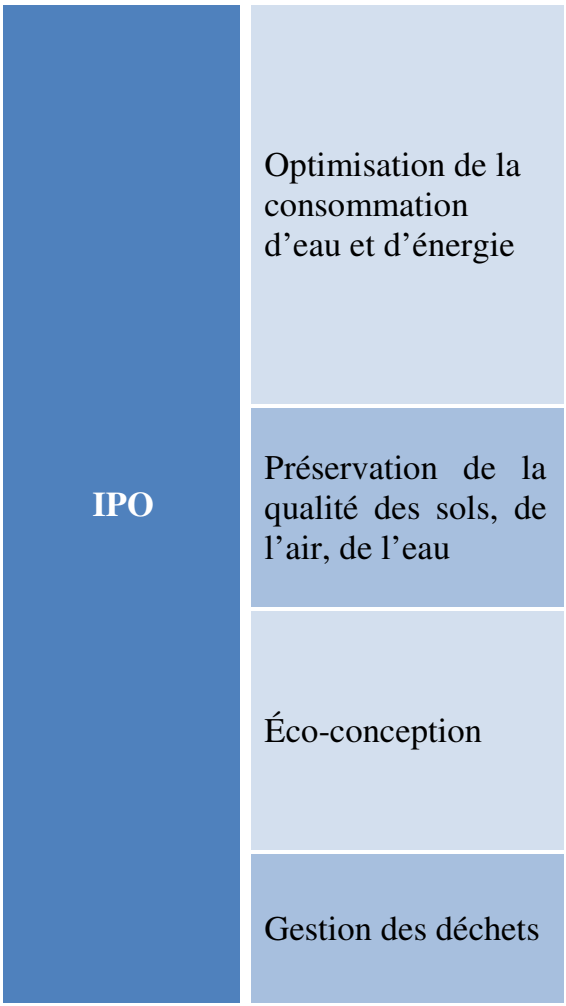

$\begin{array}{llr}\text { Évolution } & \text { de } & \text { la } \\ \text { consommation } & \text { d'eau } & \text { pour }\end{array}$

les besoins agricoles et process (en $\mathrm{m} 3$, en \%)

Évolution de la consommation d'énergie (en MWh, en \%)

Recours aux énergies renouvelables

(en \%)

Réduction des produits phytosanitaires (en \%)

Effluents traités (en \%)

Réduction des GES par bouteille produite (en \%)

Réduction du poids des emballages : allègement des Éco-conception bouteilles pour réduire les émissions de gaz à effet de serre (en tonnes équivalent $\left.\mathrm{CO}_{2}\right)$

Gestion des déchets

Déchets triés, recyclés ou valorisés (en tonne métrique, en \%) 